\title{
Research on the Biological Data Visualization Algorithm based on Self-Organizing Network
}

\author{
Xiaohan $\mathrm{Mei}^{1}$, Da Liu \\ ${ }^{1}$ School of life science, Sun Yat-sen University, Guangzhou 510275, China; \\ ${ }^{2}$ Chongqing University of Science \& Technology, Chongqing, China.
}

\begin{abstract}
Among different strategies for Artificial Neural Networks and learning calculations, Self-Organizing Feature Map (SOFM) is a standout amongst the most well-known models. The point of this study is to order features affecting the natural yield and yield of Maize utilizing Self-Organizing Feature Map calculation. In Self-Organizing Feature Map, as indicated by subjective information, the grouping propensity of yield and natural yield of Maize were researched utilizing 1000 information from 12 features. Information was gathered from the writings on the subject of Maize in google.com. Results demonstrated that when natural yield was as yield, S with pH of soil, and natural substance with grain were identified with each other nearly. Besides, grist and natural substance had nearer relationship to organic yield. At the point when Maize grain yield was yield of Self-Organizing Feature Map model, $\mathrm{S}$ with $\mathrm{pH}$ of soil, and OC with natural substance were identified with each other nearly. Generally, grist and natural substance were much nearer identified with harvest yield than different parameters. Like organic yield, marks map demonstrated that information ordered in three classes for Maize yield and the main four lines of Unified distance matrix were set in Group X. An unmistakable partition was seen among Group X with Y and Z. Our outcomes demonstrated that among the yield parts, grist was the most imperative features adding to grain yield and 500-bit weight utilizing Self-Organizing Feature Map.
\end{abstract}

Keywords: Artificial neural network; Self-organizing feature map (SOFM); Grist; Maize yield; Unified distance matrix.

\section{Introduction}

Data visualization, is a visual representation of the data for a form of science and technology research. Among them, the data of the visual form of expression is defined as, a summary in some form of information extraction, including the various properties of the corresponding information unit and variables. It is a constantly in the concept of evolution, the boundary is constantly expanding. Mainly refers to the technology more advanced technology and methods, and these techniques allow the use of graphics, image processing, computer vision and user interfaces, through expression, modeling and display of three-dimensional, surface, attributes, and animation, interpretation of data visualization. Compared with the three-dimensional modeling, such as special technology methods, data visualization method is much more widely covered by technology. Through the visual expression of biological data information, we can better result through more intuitive visual expression.

Artificial neural networks are like organic neural networks in performing capacities. It can get arrangements with improved execution contrasted and conventional strategies. It more often than not alludes to models connected in insights and artificial knowledge. Neural system models which copy the focal sensory system are a piece of hypothetical neuroscience and computational neuroscience. Among different techniques for artificial neural networks and learning calculations, Self - Organizing Feature Map is a standout amongst the most mainstream neural system models. It has a place with the class of aggressive learning networks that it is prepared utilizing unsupervised figuring out how to deliver a low-dimensional and discretized representation of the info space of the preparation tests, called a map. Self-Organizing Feature Map offers an answer for apply various perceptions connected together. At the point when a few representations are connected together, looking over them is exceptionally productive in light of the fact that they are deciphered also. The Unified distance matrix delivered from Self - Organizing Feature Map imagines distances between neighboring map units and 
in this manner demonstrates the bunch structure of the map. Tests inside the same group will be the most comparative as indicated by the variables considered. Topologically, saved mapping from contribution to yield space can give by the Self - Organizing Feature Map calculation. The Self Organizing Feature Map calculation is ideal for vector quantization. It is appropriate to numerous applications, for example, bunching, order, and information representation. Self-Organizing Feature Map has been connected as a grouping and projection calculation for high dimensional information. In another examination, the creator put forth a concentrated effort Organizing Feature Map with a specific end goal to rate incline dependability controlling variables in characteristic slants, utilized Self - Organizing Feature Map to order marine dregs. Self - Organizing Feature Map for the arrangement of water, soil and dregs quality in petrochemical locales where utilized as a part of an examination. Their outcomes indicated significant appraisal utilizing the Self - Organizing Feature Map perception capacities and highlighted zones of need that may require extra examinations furthermore gave beneficial pathway to powerful basic leadership and healing activities.

Likewise, Self - Organizing Feature Map to recognize useful gatherings was connected. For the investigation of scientists, quantitative qualities and distributional data on 87 intrusive plants in 18 areas of China were gathered to shape the grids for their study. Self - Organizing Feature Map was concentrated on, pictured and broke down of timberland stock and demonstrated that Self Organizing Feature Map played out a nonlinear dimensionality lessening and great bunching, which is a decent premise for information representation results. Specialists utilized Self - Organizing Feature Map for connections between geomorphological features of the fans and their waste bowls. The aftereffects of the investigation demonstrated that few morphologically diverse fan sorts were perceived taking into account their geomorphological attributes in the study range.

Maize is the most essential sustenance crop in different nations. Roughly, $45 \%$ of Maize developing ranges in creating nations encounter a few sorts of constraining variables, for example, abiotic stresses, supplements inadequacy and some more. Customarily, horticultural examination has concentrated fundamentally on expanding all out generation. Be that as it may, as of late, center has moved to the restricting variables in harvest generation frameworks. Specialists have just measured a predetermined number of attributes under Maize field conditions that identified with natural yield and harvest yield. It has now ended up clear that investigating countless under various field conditions can give a far reaching review of vital features in charge of Maize yield change. Considerate the significance of traits amongst vast features that are present in the dataset can assume a key part in Maize yield change. The point of the current study was determination of fundamental features identified with natural yield and Maize yield, situated in India, utilizing Self - Organizing Feature Map. It appears this is the primary explosion about grouping of the powerful limits in the natural and product harvest of Maize without anyone else's input Organizing Feature Map strategy.

\section{Related Work}

Fuzzy enrolment capacity is a successful device to uncover the relationship amongst soil and environment to foresee soil ripeness mapping. This exploration was directed to assess the ability of a joined Fuzzy Analytic Hierarchy Process technique for soil ripeness assessment of Maize in the east of Shiraz, Fars Province, India. An arrangement of enrollment capacities was directed to uncover the dirt ripeness classes, which were gotten from 64 field tests gathered through a purposive inspecting approach. Seven soil parameters: soil composition, capability of hydrogen, trade limit, nitrogen, phosphorus, potassium and natural substance of the dirt were decided for the dirt fruitfulness investigation and topical maps were created for each of these parameters with $S$ ringing strategy. With the fuzzy methodology, it is conceivable to discover humble fruitfulness ranges for Maize with worth so somewhere around 0.48 and 0.52. The consequences of the Fuzzy Analytic Hierarchy Process technique in this study demonstrated that there are areas with high richness, medium ripeness, low fruitfulness and low richness. 
The back spread artificial neural system is a prestigious and broadly practical numerical apparatus utilized for time-arrangement forecasts and approximations; which likewise characterize results for non-direct capacities. Artificial neural networks are indispensable apparatuses in the expectations of toxicant levels, for example, polycyclic fragrant hydrocarbons possibly got from anthropogenic exercises in the microenvironment. In the present work, back engendering artificial neural system was utilized as a forecast apparatus to contemplate the potential danger of PAHcarc in soils. Soil tests were gathered from different areas. It might be reasoned from this study, explanatory procedures including natural danger appraisal as utilized as a part of this study can effectively give brief forecast and source recognizable proof of significant soil toxicants.

This original copy exhibits the work with respect to the execution of neural system utilizing spiral premise capacity calculation on rapid coordinated circuit equipment portrayal dialect. It is an advanced execution of neural system. Neural Network equipment has experienced fast improvement amid the most recent decade. Not at all like the ordinary von-Neumann is design that successive in nature, Artificial Neural Networks Profit from greatly parallel handling. A substantial assortment of equipment has been intended to abuse the inalienable parallelism of the neural system models. The outspread premise capacity system is a two-layer organize whose yield units shape a direct mix of the premise capacity processed by the concealed unit and shrouded unit capacity is a Gaussian. The spiral premise capacity has a greatest of 1 when its information is 0 . As the distance between weight vector and information diminishes, the yield increments. In this manner, a spiral premise neuron goes about as a finder that produces single at whatever point the info is indistinguishable to its weight vector.

In this paper a Fuzzy Wavelet Self Organizing Map is proposed to surmised subjective complex nonlinear capacities while enhancing the estimation blunder. In this technique, the novel fuzzy wavelet strategy is joined with an unsupervised aggressive Self-Organizing Feature Map neural system. The proposed strategy utilizes two phase estimate forms: In the primary stage, guess is gotten from the Self-Organizing Feature Map neural system, a short time later in stage two with the assistance of a novel fuzzy wavelet structure, a precise and fine guess is acquired. The benefits of this new strategy are a more precise estimate and are improved system size. In the proposed technique, on the premise of Multi Resolution Analysis hypothesis, fuzzy idea and neural system parallel handling, we can achieve a superior estimation with suitable precision and utilizing a few strategies, for example, recursive slightest squares strategy, the retrogressive choice calculation and bunching thought, very exact approximations are gotten.

Execution of this proposed strategies and the customary Self - Organizing Feature Map are contrasted from three perspectives with assess the productivity of the FWSOM strategy, and recreation results outline the adequacy of this proposed technique.

In writing, there has been an expanding enthusiasm for the fuzzy surmised thinking by means of combination of neural networks along with that of the fuzzy below fuzzy neural system. Along these lines, fuzzy neural system for the most part gives a primary hypothetical premise to FAR. The majority of the current fuzzy neural system models have been projected to execute distinctive sorts of single-arranged fuzzy thinking components. The single-organized FAR, notwithstanding, is far shy of successfully taking care of convoluted basic leadership issues, prompting and developing an abnormal state of insight to take care of issues.

In this area, the vast scope of qualities saw, for the same species, can be attributed principally to: composts and water administration, for example, water administration, mineral supply and water quality, plant variables, for example, species, assortment and affectability of development stage to the anxiety and natural components, for example, atmosphere, barometrical contamination, soil composition and environmental change. The conclusion highlights the real crevice concerning WUE in the Mediterranean district. 


\section{Materials and methods}

Information gathering has been talked about here. Information exhibited in the current research was gathered from the written works on the subject of Maize in India that has happened in google.com site. A sum of 1000 information from 12 features, including watering system administration, nitrogen, phosphorus and potassium connected to the dirt ( $\mathrm{kg} / \mathrm{m} 2$ ), plant thickness (plant $/ \mathrm{m} 2$ ), developing season length that is the quantity of days, soil natural substance, $\mathrm{pH}$ of soil, precipitation sum, plant tallness in cm, grist, 500 part weight in $\mathrm{g}$, harvest file spoke to as $\mathrm{HI} \%$, organic yield spoke to as BY, $\mathrm{kg} / \mathrm{m} 2$, and grain yield spoke to as $\mathrm{kg} / \mathrm{m} 2$ were set active in Excel programming sheets. Measure of every feature that includes greatest, least, normal is computed.

Self-Organizing Feature Map has been discussed here. ANN are non-straight mapping assemblies and capable devices for demonstrating when the hidden information relationship in view of the capacity of the human mind. Artificial neural networks can decide and learn associated designs between data sets and relating target values, after which can be utilized to foresee the result of new free info information. Artificial neural networks are comparative the learning procedure of the human cerebrum and complex information regardless of the possibility that the information is loose and consequently, are in a perfect world suitable for the demonstrating of product crop, that are known for there not frequently non-direct. Artificial neural networks have extraordinary limit in prescient displaying, whereby every one of the parameters depicting the obscure circumstance can be exhibited to the prepared ANN.

Self-Organizing Map is a kind of neural system. Self-Organizing Feature Map are unsupervised artificial neural networks shaped from neurons situated on a standard, two-dimensional normal planar exhibit framework. Actually Self-Organizing Feature Map depends on unsupervised realizing, which implies that no human intercession is required amid the learning and little should be thought about the attributes of the information. Self-Organizing Feature Map offers an answer for apply various perceptions connected together. At the point when a few perceptions are connected together, looking over them is exceptionally effective on the grounds that they are deciphered also. The Unified distance matrix created from Self-Organizing Feature Map pictures distances between neighboring map units and along these lines demonstrates the bunch structure of the map. Tests inside the same bunch will be the most comparable as indicated by the variables considered.

Self-Organizing Feature Map calculation is involved two layers. In the information layer, the yield of every neurons, Wni for $\mathrm{i}$ is made equal to values like $1,2,3, \ldots$, is associated with the entire neurons of focused deposit and every association is doled out an adjustable weight, $\mathrm{vw}_{\mathrm{ij}}$ for $\mathrm{ij}=1,2,3$, and so on. Self-Organizing Feature Map calculation works as take after:

Step 1: preprocessing: in the first step to an irregular weight should be allocated to every association.

Step 2: Selection: one individual from the information space is picked.

Step 3: Corresponding: the triumphant neuron is picked when 1 is considered as the weight vector of the neuron that is present.

Step 4: Apprising: the heaviness overhaul law is made connected.

Step 5: Extension: the procedure is rehashed until a definitive objective is accomplished.

Picked information will be contrasted and all weight of associations as per the accompanying condition:

$$
W n_{I+1}=\left\{\begin{array}{c}
(1-\propto) W n_{i}+W n_{j} \\
W n_{i}
\end{array}\right.
$$

Where the champion neuron Wni, is indicated when the weight vector is nearest to info space and Wn is minimized. Where $\alpha$ is the learning rate $(0<\alpha<1)$. Factual investigations for Self-Organizing Feature Map calculation were performed utilizing Matlab programming. To start with, information was transported from Excel to Matlab and natural yield and Maize grain yield was set as yield variables and alternate variables were set as information. 


\section{Experimental Outcomes}

Self-Organizing Feature Map for natural yield: The representation for natural yield as yield Unified distance matrix in the upper left. As indicated by study, the distinctive gathered statistics were connected by situation. In every figure, hexagon in a specific spot related to the same map unit. The legend for each of the hexagons demonstrated level shading contrasted with each other. In Self-Organizing Feature Map strategy, comparative hues indicated straight association amongst the parameters. It has been noted that $\mathrm{S}$ is associated to $\mathrm{pH}$ of soil, Irrigation administration with 500-bit weight along with natural substance with grist were identified with each other nearly. Additionally, grist and natural substance had nearer relationship to organic yield than different parameters. Conversely, $\mathrm{pH}$ of soil, spike/m2 and harvest file had negative connections to natural yield.

On point of interest contemplating, pen down in black and white hexagons were information that has consumed by every hubs in the neural system. As indicated by the most extreme number of hexagons was 5, demonstrating that the greatest information for these zones was 5 . Then again, the base amount of hexagons is considered as 0 , showing that these territories had no information. Likewise, the information obtained had high thickness in reality information had great conveyance. Names map demonstrated that information characterized as tri modules for organic crop and the main quad columns of Unified distance matrix was as gathering X. Alternate gatherings were $\mathrm{Y}$ and $\mathrm{Z}$ structure and there was clear partition between gathering $\mathrm{X}$ and gathering $\mathrm{Y}$ in the Unified distance matrix of marks. The attributes of every gathering identified with organic yield that controlled by mark map gave. Since the information from cluster 1 to cluster 2 demonstrated by expanding $\mathrm{S}$ from that of 0.004 to $0.026(\mathrm{~kg} / \mathrm{m} 2)$, plant thickness developing season from 26.7 to 28.9 (d) the most astounding organic yield $(2.807 \mathrm{~kg} / \mathrm{m} 2)$ was seen in group 2 .

A scientist reported that field water status, for example, watering system administration or precipitation, was another imperative feature identified with organic yield, and S connected to the dirt worth was not observed to be critical utilizing property weighting models. In our concentrate, no solid relationship was seen amongst $S$, and watering system administrations with organic yield.

It was found that grist and plant tallness was connected to organic yield, decidedly. As of late, an exploration demonstrated that nitrogen connected to the dirt, a key component in harvest nourishment had a critical part in expanding natural harvest and Maize grain harvest. Strikingly, no solid connections were seen amongst $\mathrm{N}$ connected and plant stature along with natural harvest though expanding natural substance identified with organic yield change. The considered characteristics of the two clusters, as Irrigation, 43.6 in cluster 1 and 65.1 in cluster 2. The amount of rainfall in cluster 1 shows $445.5 \mathrm{~mm}$ and $757.4 \mathrm{~mm}$ in cluster 2 . The $\mathrm{pH}$ of soil in 7.4 of cluster 2 roughly equals to the 7.6 of cluster 2 .

Self-Organizing Feature Map for Maize yield has been discussed in this section. Rendering figure 1, the architecture of Self-organizing feature map (SOFM) is seen. Likewise, negative connections were seen amongst $\mathrm{S}$ and $\mathrm{pH}$ of soil with harvest. Inquisitively, no optimistic association was seen amongst harvest Index and 500 bit weight with harvest. By and large, grist and natural substance were much nearer identified with harvest yield than different parameters. Name map verify that study information arrange in two groups for harvest yields. Like organic yield, marks map demonstrated that information characterized in three gatherings for Maize yield and the main four columns of Unified distance matrix was as gathering X. In like manner, an unmistakable detachment was seen between gathering $\mathrm{X}, \mathrm{Y}$ and $\mathrm{Z}$ in the Unified distance matrix.

Information attributes of every gathering for determination of Self-Organizing Feature Map calculation. Plainly bunch 2 that demonstrate the most elevated yield than group1 with the harvest index of $33.1 \%$ to $55.1 \%$. Likewise, Maize yield was not influenced by plant thickness, developing season, $\mathrm{pH}$ of soil, and 500-piece weight. Scientist reported that in view of administered feature choice model, natural substance and precipitation sum influenced Maize grain yield, while $\mathrm{pH}$ of soil marginally affected Maize grain yield. They additionally showed that variables arrangement utilizing feature choice calculation might be an appropriate choice for deciding the vital elements adding to Maize grain yield, and for giving an extensive perspective of various attributes. Utilizing 
unsupervised weighting calculations, different characteristic weighting calculations, harvest list was less imperative in present day Maize genotypes and was just chosen.

Then again, Maize grain yield might be expanded by enhancing biomass for any type of yield record in three winter Maize populaces, this has been concentrated on. Reported that enhancing harvest record has all the earmarks of being troublesome, and late increments in Maize harvest have been credited to increments in grist. The outcomes got demonstrates that among the yield segments, grist was the most vital features adding to harvest than spike $/ \mathrm{m}^{2}$ and weight is 500 bit.

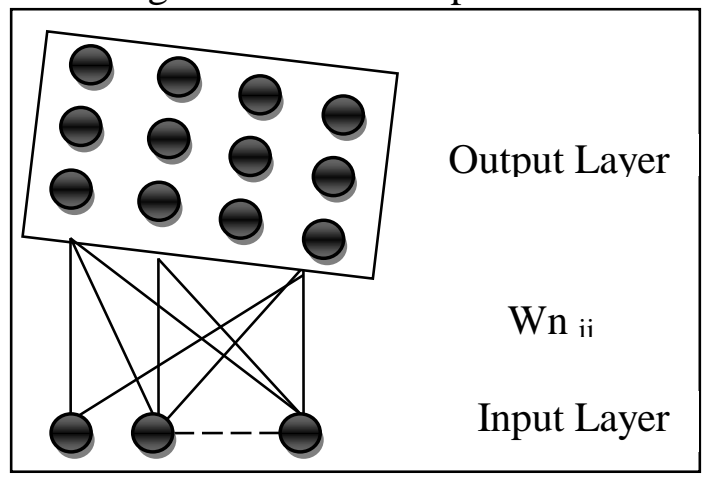

Figure 1. Self-organizing feature map (SOFM)-the architecture

In comparative study on grain, soil, natural substance, electrical conductivity of water, harvest file, and plant thickness had the minor impact on grain yield. Whatever remains of the features including 500-portion weight, soil composition, plant tallness, $\mathrm{pH}$ of soil, and potassium and phosphorus connected to the dirt were perceived to be insignificant.

\section{Conclusion}

The outcomes demonstrated that the SOFM is an amazing instrument in representation of high dimensional information. In that capacity Self-Organizing Feature Map technique is most appropriate for information understanding period of the learning disclosure process. Self-Organizing Feature Map technique comprised of Unified distance matrix and mark. Utilizing of the Unified distance matrix indicates rich group that in the names by lines shading. Unified distance matrix demonstrated that a portion of the information have firmly identified with each other. Extra utilizing projection discovers thickness of information. The current research on the demonstration of the PC projection had high thickness for natural and harvest. Generally, the essential features identified with natural yield and grain yield change were grist and OC. Interestingly, our outcomes demonstrated that Self-Organizing Feature Map can give an extensive perspective of essential features add to Maize grain harvest change and there are degree by choosing the appropriate cultivars with an advanced grist. The research leads to outlook in Maize generation and led to discovery of the principle components adding to natural yield and Maize harvest from various Maize arena without anyone else's input Organizing Feature Map.

\section{References}

[1]. Bijanzadeh E, Mokarram M (2013) the use of fuzzy- AHP methods to assess fertility classes for Maize and its relationship with soil salinity: east of Shiraz, Iran: a case study. Aust J Crop Sci. 7:1699-1706.

[2]. Bijanzadeh E, Emam Y, Ebrahimie E (2010) Determining the most important features contributing to Maize grain yield using supervised feature selection model. Aust J Crop Sci. 4: 402-407.545

[3]. Bijanzadeh E, Emam Y, Ebrahimie E, Ebrahimie M (2012) Application of unsupervised weighting algorithms for identifying important attributes and factors contributing to grain and biological yields of Maize. Crop Breed J. 2:111-117. 
[4]. Bijanzadeh E, Naderi R (2014) Application of supervised and unsupervised algorithms to find the important features related to barley (Hurdeum vulgare L.) grain yield: a new vista in data mining. Aust J Crop Sci. 8:1590-1596.

[5]. Dhubkarya DC, Nagariya D, Kapoor R (2010) Implementation of a radial basis function using by VHDL. Global J Com Sci Tech. 21:16-19. 